\title{
Non-invasive tools for detection of liver disease in children and adolescents with cystic fibrosis
}

\author{
Antonella Tosco ${ }^{1}$, Angela Sepe ${ }^{1}$, Alice Castaldo ${ }^{1}$, Andrea Catzola ${ }^{1}$, Chiara Cimbalo ${ }^{1}$, Valentina Angelini ${ }^{2}$, \\ Gianfranco Vallone ${ }^{3}$, Roberto Buzzetti ${ }^{4}$, Valeria Raia ${ }^{1}$, Maria Grazia Caprio ${ }^{5}$ \\ ${ }^{1}$ Pediatric Unit, Department of Translational Medical Sciences, Regional Cystic Fibrosis Center, Federico II University of Naples, Naples, Italy; \\ ${ }^{2}$ Department of Oncoematologia, Diagnostica per immagini e morfologica e Medicina Legale, University of Naples Federico II, Naples, Italy; \\ ${ }^{3}$ Department Life and Health "V. Tiberio", University of Molise, Campobasso, Italy; ${ }^{4}$ Freelance Epidemiologist, Bergamo, Italy; ${ }^{5}$ Institute of \\ Biostructure and Bioimaging IBB, CNR, Naples, Italy \\ Contributions: (I) Conception and design: A Tosco, MG Caprio; (II) Administrative support: C Cimbalo; (III) Provision of study materials or patients: \\ MG Caprio, V Raia, A Tosco, A Sepe; (IV) Collection and assembly of data: A Castaldo, A Catzola, C Cimbalo; (V) Data analysis and interpretation: \\ R Buzzetti, V Raia, G Vallone; (VI) Manuscript writing: All authors; (VII) Final approval of manuscript: All authors. \\ Correspondence to: Antonella Tosco. Pediatric Unit, Department of Translational Medical Sciences, Regional Cystic Fibrosis Center, Federico II \\ University of Naples, Naples, Italy. Email: antonella.tosco@unina.it.
}

Background: Cystic fibrosis (CF) is a multi-organ genetic disease caused by mutations in the cystic fibrosis transmembrane conductance regulator (CFTR) gene which encodes the CFTR protein. CF-associated liver disease (CFLD) is a common complication; diagnosis is based on clinical, laboratory findings and abdominal imaging. However, non-invasive diagnostic approaches are needed to early detect CFLD, its progression and severity. Recent studies demonstrate a possible role of point shear wave elastography (p-SWE) with liver stiffness measurement (LSM) as a tool for CFLD diagnosis also in children. This non-invasive technique measures liver stiffness to assess liver fibrosis and is suggested to be less operator-dependent compared to ultrasonography. Aim of our prospective observational study is to investigate the role of p-SWE with LSM for CFLD diagnosis in children and adolescents with CF and to compare this finding with aspartate aminotransferase to platelet ratio index (APRI), fibrosis index based on four factors (FIB-4) and gammaglutamyl-transpeptidase to platelet ratio (GPR) indices.

Methods: Fifty-nine children with CF, who had routinely undergone abdominal imaging, were consecutively enrolled. Laboratory findings and clinical data were recorded, as abdominal ultrasound and shear wave elastography at baseline. The cases were divided into two groups based on collected data and classified as CFLD and CFnoLD (without liver disease) according to Debray criteria. APRI, FIB-4 and GPR fibrosis indices were also evaluated.

Results: Twenty-four/59 (40.7\%) were defined as CFLD. LSM test is superior to the APRI $(\mathrm{P}<0.001)$, the FIB-4 test $(\mathrm{P}=0.001)$ and the GPR test for early detection of liver fibrosis. LSM had an area under receiver operating characteristic $($ ROC) curve $=0.818$ (95\% CI: 0.702-0.934) compared with APRI $(0.571,95 \%$ CI: 0.421-0.722), FIB-4 (0.656, 95\% CI: 0.511-0.801) and GPR (0.632, 95\% CI: 0.485-0.779). At a cut-off of $\geq 6.2$ LSM show a sensitivity of $75.0 \%$ and a specificity of $88.6 \%$.

Conclusions: LSM by transient p-SWE is a non-invasive, highly accessible, reliable, and reproducible test that can be used to assess early detection of liver fibrosis and its severity in children and adolescents with CF, limiting the use of liver biopsy. These preliminary observations point to the need of larger study population to confirm our data.

Keywords: Cystic fibrosis (CF); liver disease; ultrasound; hepatic stiffness

Submitted Feb 20, 2021. Accepted for publication Aug 12, 2021.

doi: $10.21037 /$ tp-21-68

View this article at: https://dx.doi.org/10.21037/tp-21-68 


\section{Introduction}

Cystic fibrosis (CF) is one of the most common inherited disease in the Caucasian population (1). The multiorgan involvement of disease may include a wide range of liver abnormalities, known as cystic fibrosis-associated liver disease (CFLD) (2). In the last decades as CF patient's life expectancy has improved the prevalence of CFLD has increased ranging from $5.7 \%$ to $39 \%(3,4)$. Usually, CFLD starts at the end of the first decade of life (4) with a progression to multilobular cirrhosis and in a $5 \%$ of cases to chronic liver failure that may occur even in paediatric population. Hepatobiliary disease is the fourth leading cause of mortality in CF patients $(5,6)$. It is well known that CFLD represents a risk predictor of an unfavourable evolution and prognosis of CF $(7,8)$. Therefore, noninvasive diagnostic approaches to early detect CFLD, its progression and severity are needed. Over the past years, new techniques to diagnose features of CFLD have been investigated. Although most of these tests confirm CFLD involvement, they are not still suitable to distinguish different phenotypical presentations or predict progression to clinically relevant cirrhosis or portal hypertension.

To date CFLD is diagnosed according to EuroCare CFLD criteria, which include $\geq 2$ of the following: persistent abnormal levels of liver function tests over 12 months, clinical hepatomegaly and/or splenomegaly, or ultrasound evidence of liver disease (7). Current methods to detect CFLD include clinical examination, monitoring of liver enzymes, ultrasonography, abdominal computed tomography, and abdominal magnetic resonance imaging. Despite hepatic biopsy procedure is still considered the gold standard for the diagnosis of CFLD, in paediatric population it is considered invasive and difficult to perform. For this reason, the abdominal ultrasound test is widely used to detect CFLD in children with CF, however it is limited by inter-observer variability and poor sensitivity and specificity (7).

As currently no factors are being identified to provide a guidance for selecting children at risk of chronic liver disease, the potential of combined markers for early detection of liver fibrosis must be explored. The use of conventional biomarkers can potentially meet the need for an inexpensive, reproducible, sensitive screening tool to predict histology in CFLD. Among biomarkers, aspartate aminotransferase to platelet ratio index (APRI), previously studied in children infected with hepatitis $B$, $\mathrm{C}$ and biliary atresia (9-11) as a non-invasive marker of fibrosis and cirrhosis and fibrosis index based on the four factors (FIB-4), previously studied in adults with hepatitis $\mathrm{C}$ and nonalcoholic fatty liver disease, have been used to predict degree of fibrosis in paediatric CFLD (12-16). The gamma-glutamyl-transpeptidase to platelet ratio (GPR) is a new serum diagnostic model for liver fibrosis and cirrhosis, for which there are conflicting data about its superiority over APRI and FIB-4 in diagnosing significant fibrosis and cirrhosis in patients with chronic hepatitis B (17); few data exist in CF. Moreover, recent studies demonstrate a possible role of transient elastography (TE) with liver stiffness measurement (LSM) as a tool for CFLD diagnosis also in children $(12,14,17-20)$. The ultrasound elastography techniques allow a non-invasive method to estimate the degree of liver fibrosis. TE is a non-imaging elastographic technique, while point shear wave elastography (p-SWE) and 2D-SWE combine imaging with elastography. The evidence currently suggests that $\mathrm{p}-\mathrm{SWE}$ is as accurate as but more reliable than TE, while 2D-SWE is more accurate than TE (21-23). Some studies also speculated that the combination of TE and APRI could increase the accuracy of CFLD detection $(12,24)$.

The aim of this study is to investigate the role of p-SWE for CFLD evaluation in a cohort of subjects with CF and its diagnostic performance to assess liver disease progression risk. We also investigated the usefulness of LSM, APRI, FIB-4 and GPR as non-invasive tools for the diagnosis of CFLD.

We present the following article in accordance with the STARD reporting checklist (available at https://dx.doi. org/10.21037/tp-21-68).

\section{Methods}

A prospective observational study was performed including children and adolescents with diagnosis of CF. Fiftynine subjects with CF were consecutively enrolled from April 2018 to April 2019 at the Regional Paediatric Centre of University Federico II in Naples. All enrolled patients underwent abdominal ultrasound and shear wave elastography at baseline. Liver p-SWE and 2D-SWE were performed by a single/two trained operator(s), blinded to patients' clinical information, at the Paediatric Radiology Section of the University Hospital. A $60 \%$ success rate and an interquartile range (IQR) of less than $30 \%$ of the mean value of LSMs were considered reliable (25).

Measurements of liver stiffness were performed using a Samsung RS85A ultrasound scanner with Prestige 
equipment (Samsung Medison Co. Ltd., Seoul, Korea), with a convex array transducer (CA1-7; Samsung Medison Co. Ltd., Seoul, Korea). The smart-shear wave elastography (S-SWE), installed on this ultrasound scanner, is a particular type of p-SWE. In this technique, a localized transient displacement is generated using an acoustic radiation force impulse (ARFI). Some studies have demonstrated a potential role for ARFI in identifying liver disease even in children $(26,27)$. It creates a transient shear wave spreading with cylindrical symmetry away from the pushingbeam's axis and focus, which is strongest at the depth of the pushing-beam's focus (22). The shear displacement propagates along the ultrasound imaging beam, so the small displacements of the shear wave are measured and its time of arrival at lateral positions of the region of interest (ROI) is detected (22). The S-SWE measurements were carried out in the morning, after at least 6-hour fasting time. For the examination, all the children were placed in a supine position, with the right arm under the head. The ROI, from which the shear wave velocities were measured, was placed in the right hepatic lobe and the radiologist first localized the best acoustic window as an area without large vessels and with a minimum thickness of the hepatic parenchyma of $6 \mathrm{~cm}$ at $1.5 \mathrm{~cm}$ to the Glissonian. The probe has been placed on the chest wall at the intercostal space level next to the right hepatic lobe, on the mid-axillary line. B-mode imaging was used to confirm the correct placement of the transducer.

S-SWE enables to place freely the ROI with a fixed height of $1 \mathrm{~cm}$. The width is automatically adjusted depending on the measurement depth. The method had an only performance index, called "Reliability Measurement Index" (RMI), calculated by the weighted sum of the residual of the wave equation and the magnitude of the shear wave. RMI range is from -1.0 to 1.0 and, in characterizing diffuse liver disease, a standardized value of 0.5 or higher is considered acceptable and correlates with reproducible measurements, according to the manufacturer (22). This value is used to filter out unreliable measurements and results in performance improvement of shear wave elastography. The average value was considered representative of liver elasticity and therefore of its rigidity. For each patient, 10 measurements were performed in succession validated by the same device, with the result expressed in kilopascal (kPa).

Liver function tests including aspartate aminotransferase (AST), alanine aminotransferase (ALT), gamma glutamyl transferase (GGT), alkaline phosphatase (ALP) and platelet count were assessed at enrolment; parameters were analysed using validated assays at the centralized laboratory, the same day of LSM. Clinical features were also recorded, including body mass index (BMI), forced expiratory volume in 1 second (FEV1) and maximum average expiratory flow (MMEF), expressed as a percentage of the predicted value by age, sex and ethnicity, and measured by spirometry performed only in collaborating patients. Furthermore, data about chronic colonization and the presence of comorbidity were collected. In particular, chronic colonization by pseudomonas aeruginosa (PA), was defined as the positivity of at least 3 consecutive sputa in 6 months; the presence/ absence of diabetes or carbohydrate intolerance was assessed on the basis of oral glucose tolerance test (OGTT). Treatments with ursodesossicolic acid and probiotics assumption, as well as the use of new therapies, were also recorded. Two groups were classified as CFLD (with liver disease according to Debray criteria) and CFnoLD (without liver disease). In particular, the patients were clinically defined as CFLD in presence of at least 2 of the 3 signs or symptoms: (I) hepatomegaly \pm splenomegaly; (II) persistent elevation ( $>6$ months) of serum ALT (ALT $>1.5 \times$ upper limit of normal); (III) abnormal ultrasound scan (showing all the abnormal echogenicity or nodularity that suggests the presence of cirrhosis) (7). APRI, FIB-4 and GPR fibrosis index were calculated, as previously described, by the statistician blinded to the clinical information of patients (13):

- APRI $=[$ AST/normal upper limit of AST (U/L) $\times 100] /$ platelet count $\left(10^{9} / \mathrm{L}\right)$;

* FIB-4 = [age (years) $\times$ AST $(\mathrm{U} / \mathrm{L})] /\left[\right.$ platelet count $\left(10^{9} / \mathrm{L}\right)$ $\times \sqrt{ } \operatorname{ALT}(\mathrm{U} / \mathrm{L})]$

* GPR = [GGT (U/L)/normal upper limit of GGT)/ platelet count $\left(10^{9} / \mathrm{L}\right) \times 100$.

An AST value of $34 \mathrm{U} / \mathrm{L}$ was used as the upper limit of the normal value.

Patients with chronic hepatobiliary diagnosed for other causes were excluded.

The study was conducted in accordance with the Declaration of Helsinki (as revised in 2013). The approval of ethic committee was not obtained because the data were anonymous, and the analysed procedures are carried out as part of the routine follow-up of patients without any additional risk. Before all study procedures, all patients and/ or their parents signed an informed consent form.

\section{Statistical analysis}

After a descriptive analysis of the distributions of the 
different variables (calculation of means, standard deviations, proportions) the association between each variable and the outcome (prevalence of CFLD according to Debray criteria) were tested through a series of univariate analyses, with Student's $t$-test on the means for numerical variables and with the exact Fisher's test for the proportions. Statistical significance was tested with alpha $=0.05$; the variables that were significantly associated $(\mathrm{P}<0.05)$ with the presence of liver disease were then studied in a multiple logistic analysis model, to estimate the diagnostic performances of the LSM test and the APRI, FIB-4, GPR, AST, ALT, GGT, ALP, age and BMI tests. Sensitivity, specificity, predictive values, likelihood ratios and the area under receiver operating characteristic (ROC) curve (AUC) were calculated, with the limits of the $95 \%$ confidence interval (CI) for all these parameters. Through ROC analysis the optimal cut-off, that maximizes the sum of true positives and true negatives values (Youden's Index), was estimated.

Hanley et al. test (28) was applied for the comparison between the LSM test and the other three tests. Finally, through a multiple logistic regression model, we tested the independent contribution of the three LSM tests, APRI and FIB-4 for the diagnosis of liver disease, to understand whether the diagnostic performance of the LSM test could be further improved including other two tests performed simultaneously.

Since this is a study with exploratory purposes, a formal calculation of the sample size was not carried out and an attempt was made to recruit all possible subjects affected by CFLD. Despite this limited number of subjects available (given the rarity of the condition investigated), we considered that with 25 subjects with CFLD we would have obtained the following $95 \%$ confidence limits for sensitivity: from $64 \%$ to $93 \%$ in case of sensitivity $=80 \%$; from $80 \%$ to $99 \%$ in case of sensitivity $=90 \%$. Similarly, the following $95 \%$ CI limits for specificity could be obtained with 45 control subjects: $68 \%$ to $89.4 \%$ in case of specificity $=80 \%$; $88.8 \%$ to $96.2 \%$ in case of specificity $=90 \%$. With these sample sizes, even a difference of 0.15 (e.g., 0.80 vs. 0.65 ) in the AUC between new and old tests would be appreciated as significant (alpha $=0.05)(29)$.

\section{Results}

Fifty-nine patients (31 females) diagnosed as CF were enrolled in the study, with an average age of (mean \pm SD) $9.24 \pm 4.55$ years, range $1-17.8$ years. In all 59 patients CF was diagnosed based on 2 causing mutations and sweat tests with chloride concentrations above $60 \mathrm{mEq} / \mathrm{L}$. In total $51 / 59(86.4 \%)$ patients had pancreatic insufficiency and $5 / 59(8.5 \%)$ patients had a history of recurrent pancreatitis. According to the described criteria 24/59 (40.7\%) were defined as CFLD. Gallbladder was not visible in 4 patients and in 3 patients only a draft was visible. All the patients classified as CFLD, except one, had two severe mutations in the CFTR gene. Demographic and clinical characteristics of study patients were collected from medical records and are listed in Table 1.

The variables significantly associated with CFLD at univariate analysis were studied with multiple logistic regression model, having CFLD as a dependent variable. Given the small subject number, different models were evaluated including subsets of the above variables.

Table 2 showed the comparison between the diagnostic performance of non-invasive tools.

By applying appropriate statistical tests (28), we reported that the LSM test is superior to both the APRI test ( $\mathrm{z}$ $=2.89, \mathrm{P}<0.001)$ and the FIB- 4 test $(\mathrm{z}=2.28, \mathrm{P}=0.001)$. The value of the linear correlation coefficient between LSM and APRI and between LSM and FIB-4 are 0.34 and 0.52, respectively. The logistic regression analysis showed that the addition of the other tests (APRI and/or FIB-4) to LSM do not add any significant contribution to the diagnosis (their independent effect is not significant, data not shown). Finally, a multiple regression model including LSM, age and pancreatic insufficiency showed that age and pancreatic insufficiency do not have a significant effect, additional to LSM, on the risk of liver disease (data not shown).

No adverse events were recorded during tests execution.

\section{Discussion}

CFLD is an umbrella term that encompasses a wide spectrum of liver disease including elevated liver enzymes, hepatic steatosis, neonatal cholestasis, focal biliary cirrhosis, multilobular cirrhosis, and cholangiopathy (13). In 2007, the North American Cystic Fibrosis Foundation classified CFLD into three categories: pre-clinical disease, CFLD without cirrhosis and portal hypertension, and CFLD with cirrhosis and portal hypertension. The most clinically relevant forms of CFLD are biliary cirrhosis and portal hypertension because of their associated mortality risk. Within the first decade of life up to $10 \%$ of children with CFLD can develop CF from focal biliary cirrhosis to multilobular cirrhosis and portal hypertension.

As CFLD represents the most common extrapulmonary 
Table 1 Clinical characteristics of CFLD vs. CFnoLD

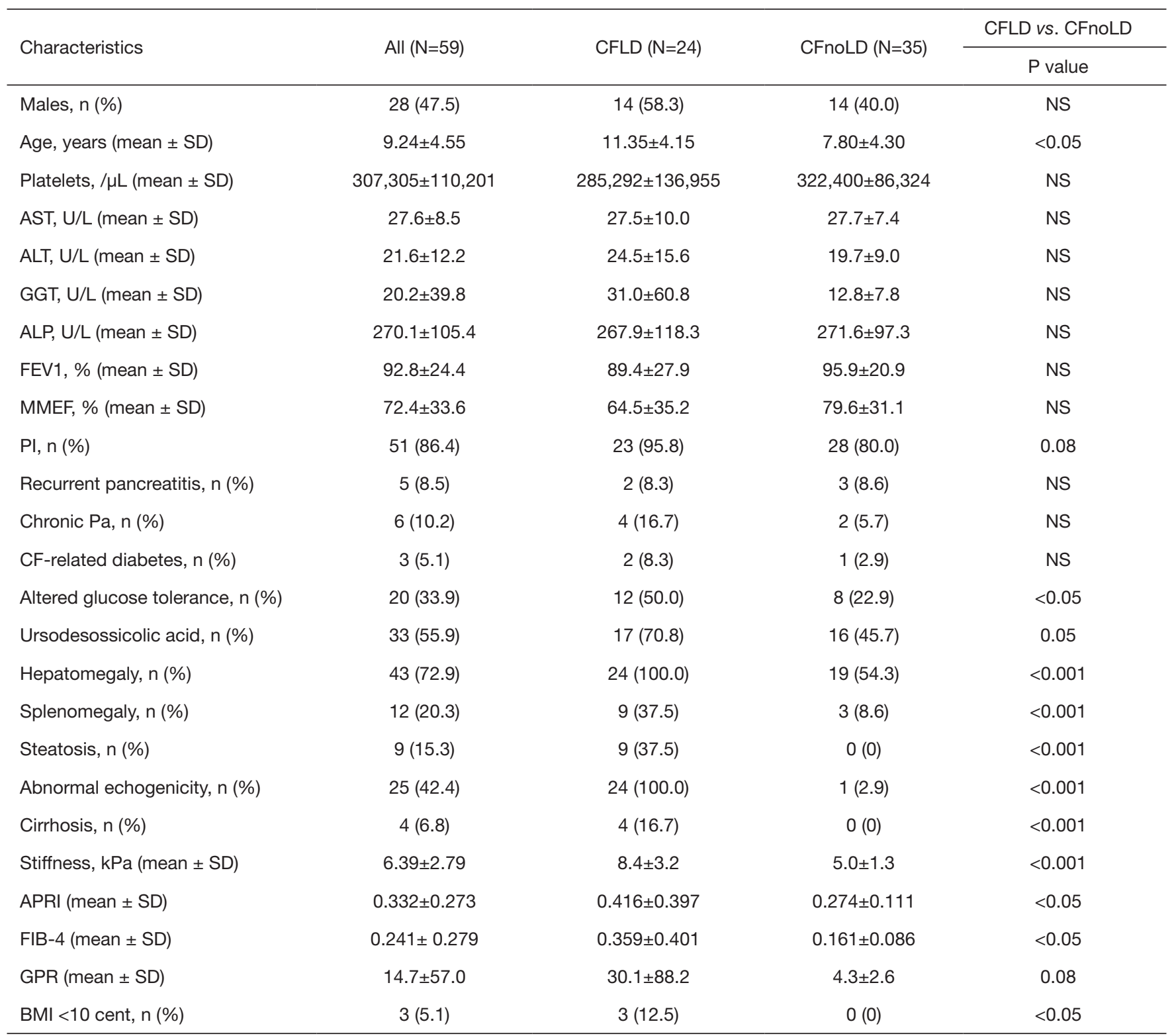

ALP, alkaline phosphatase; ALT, alanine aminotransferase; APRI, aspartate aminotransferase to platelet ratio index; AST, aspartate aminotransferase; BMI, body mass index; CF, cystic fibrosis; CFLD, cystic fibrosis-associated liver disease; CFnoLD, cystic fibrosis without liver disease; GGT, gamma glutamyl transferase; GPR, gamma-glutamyl-transpeptidase to platelet ratio; FEV1, forced expiratory volume in 1 second; FIB-4, fibrosis index based on four factors; MMEF, maximum average expiratory flow; N, number; NS, not significant; $\mathrm{Pa}$, Pseudomonas aeruginosa; PI, pancreatic insufficiency.

cause of morbidity and mortality in CF patients, an early diagnosis of CFLD is urgently needed. Although defined criteria for the diagnosis and management of CFLD have been recently published, they might miss early stages of CFLD, especially in the absence of significant fibrosis deposition within the liver. In CF subjects an early marker of liver fibrosis continues to pose a great challenge, as no single diagnostic modality except for the liver biopsy has demonstrated sensitivity and specificity values such enough to predict disease risk. Current recommendations include to perform at least annually biochemical blood tests and abdominal ultrasound which however have limited 
Table 2 Comparison of non-invasive tools

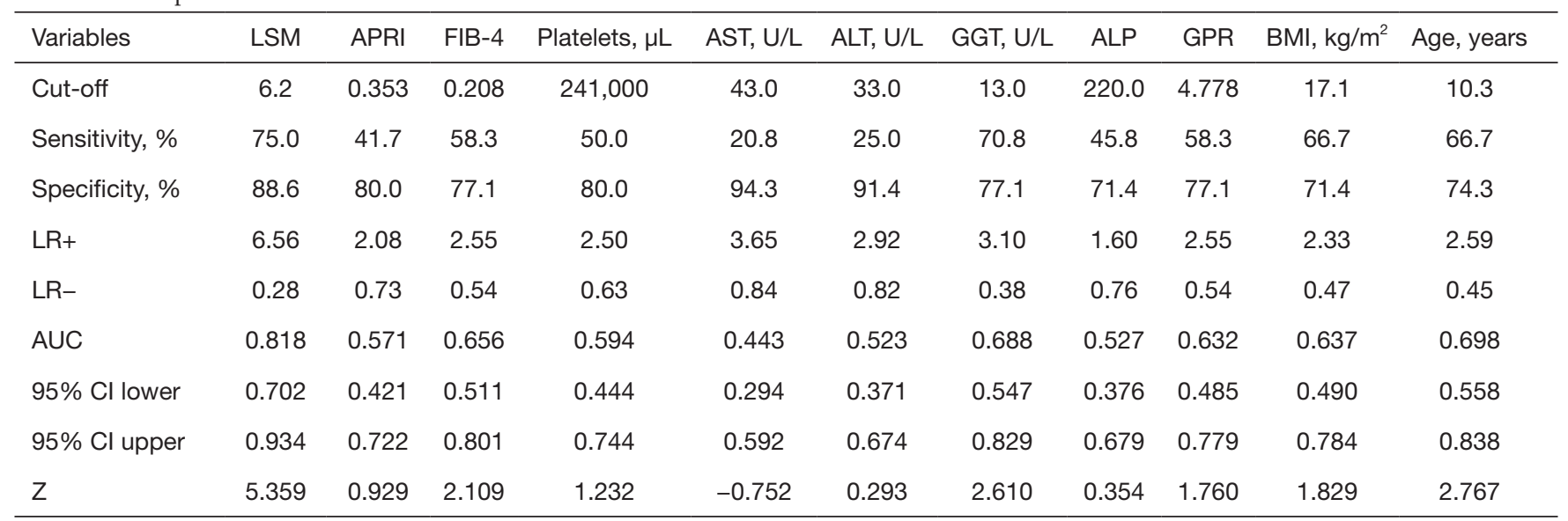

ALP, alkaline phosphatase; ALT, alanine aminotransferase; APRI, aspartate aminotransferase to platelet ratio index; AST, aspartate aminotransferase; AUC, area under receiver operating characteristic curve; BMI, body mass index; 95\% Cl, 95\% confidence interval; FIB4, fibrosis index based on four factors; GGT, gamma glutamyl transferase; GPR, gamma-glutamyl-transpeptidase to platelet ratio; LR, likelihood ratios; LSM, liver stiffness measurement.

sensitivity and specificity (7). In case of uncertain diagnosis, it is indicated to perform a liver biopsy that has a higher morbidity risk in children and requires the use of a general anaesthesia which can further complicate lung disease. The early progression of liver disease in cirrhosis in selected cases suggests identifying earlier and more accurate markers of liver fibrosis in order to anticipate therapeutic intervention and follow-up. Non-invasive diagnostic methods are therefore necessary for the detection of CFLD and to define a grading for severity of this complication. In previous study APRI appears to be superior to the FIB4 in the assessment of fibrosis. An APRI score $>0.264$ had a sensitivity of $73.1 \%$ and specificity of $70.2 \%$ for predicting CFLD (13). FIB-4, however, performed better when differentiating patients with portal hypertension from those without portal hypertension, with an AUC of 0.91 and a sensitivity and specificity of $78 \%$ and $93 \%$ respectively (13). Stonebraker et al. have also shown significantly different FIB-4 and APRI scores among patients with and without varices (29). The inclusion of biomarkers, such as APRI and FIB-4, into the diagnostic criteria for CFLD has demonstrated to capture more patients with liver involvement at an early stage compared to the Debray criteria and warrants further consideration given their ability to identify patients with both fibrosis and portal hypertension (30).

In our study, we speculate that new non-invasive tools may detect liver function in CF, such as the APRI and FIB4 indices and the LSM values $(12,13)$ in a cohort of children and adolescents with CF.

Main results provided that LSM values measured by p-SWE and 2D-SWE are able to discriminate CFLD from CFnoLD. As previously reported, the measurement of liver stiffness (LSM) has a high diagnostic accuracy for the detection of CFLD in adults and children. The optimal cut-off of LSM over $6.2 \mathrm{kPa}$ has shown to get a high sensitivity and specificity (14), while in our study APRI, FIB-4 and GPR indices had a poor diagnostic capacity for the best cut-offs respectively $\geq 0.353, \geq 0.208$ and $\geq 4.778$, differently from published data $(13,31)$. Indeed, LSM values were better than the clinical markers APRI, FIB-4 and GPR in discriminating CFLD patients in a cohort of children/adolescents with CF with different stages of liver disease.

A potential advantage of elastographic measurement over liver biopsy is the ability to monitor liver disease progression (32). Recent studies have shown that shear wave elastography is an excellent method of assessing the degree of severity of liver fibrosis not only in adults but also in children and adolescents $(23,33,34)$.

Based on these preliminary results we speculate that the measurement of the "stiffness" for evaluating hepatic liver tissue by p-SWE and 2D-SWE may be used for the early detection of liver fibrosis. p-SWE and 2D-SWE are noninvasive, highly accessible, reliable, and reproducible test that can be used to screen liver disease and to assess the severity of fibrosis in children with CF limiting the use of liver biopsy. Our preliminary observations point to the 
need of larger study population to confirm results.

\section{Acknowledgments}

Funding: None.

\section{Footnote}

Reporting Checklist: The authors have completed the STARD reporting checklist. Available at https://dx.doi. org/10.21037/tp-21-68

Data Sharing Statement: Available at https://dx.doi. org/10.21037/tp-21-68

Conflicts of Interest: All authors have completed the ICMJE uniform disclosure form (available at https:// dx.doi.org/10.21037/tp-21-68). RB reports contract from Department of Translational Medical Sciences of the University of Naples Federico II. The other authors have no conflicts of interest to declare.

Ethical Statement: The authors are accountable for all aspects of the work in ensuring that questions related to the accuracy or integrity of any part of the work are appropriately investigated and resolved. The study was conducted in accordance with the Declaration of Helsinki (as revised in 2013). The approval of ethic committee was not obtained because the data were anonymous, and the analysed procedures are carried out as part of the routine follow-up of patients without any additional risk. Informed consent was taken from all patients or their parents.

Open Access Statement: This is an Open Access article distributed in accordance with the Creative Commons Attribution-NonCommercial-NoDerivs 4.0 International License (CC BY-NC-ND 4.0), which permits the noncommercial replication and distribution of the article with the strict proviso that no changes or edits are made and the original work is properly cited (including links to both the formal publication through the relevant DOI and the license). See: https://creativecommons.org/licenses/by-nc-nd/4.0/.

\section{References}

1. Ratjen F, Bell SC, Rowe SM, et al. Cystic fibrosis. Nat Rev Dis Primers 2015;1:15010.

2. Kamal N, Surana P, Koh C. Liver disease in patients with cystic fibrosis. Curr Opin Gastroenterol 2018;34:146-51.

3. Kobelska-Dubiel N, Klincewicz B, Cichy W. Liver disease in cystic fibrosis. Prz Gastroenterol 2014;9:136-41.

4. Colombo C. Liver disease in cystic fibrosis. Curr Opin Pulm Med 2007;13:529-36.

5. Cystic Fibrosis Foundation. Patient registry. Annual data report 2018. Available online: https://www.cff.org/ Research/Researcher-Resources/Patient-Registry/2018Patient-Registry-Annual-Data-Report.pdf. Accessed September 10, 2020.

6. European Cystic Fibrosis Society. Patient Registry. Annual data report 2017. Available online: https://www.ecfs. $\mathrm{eu} /$ sites/default/files/general-content-images/workinggroups/ecfs-patient-registry/ECFSPR_Report2017_ v1.3.pdf. Accessed September 10, 2020.

7. Debray D, Kelly D, Houwen R, et al. Best practice guidance for the diagnosis and management of cystic fibrosis-associated liver disease. J Cyst Fibros 2011;10 Suppl 2:S29-36.

8. van de Peppel IP, Bertolini A, Jonker JW, et al. Diagnosis, follow-up and treatment of cystic fibrosis-related liver disease. Curr Opin Pulm Med 2017;23:562-9.

9. Grieve A, Makin E, Davenport M. Aspartate Aminotransferase-to-Platelet ratio index (APRi) in infants with biliary atresia: prognostic value at presentation. J Pediatr Surg 2013;48:789-95.

10. Xu XY, Kong H, Song RX, et al. The effectiveness of noninvasive biomarkers to predict hepatitis B-related significant fibrosis and cirrhosis: a systematic review and meta-analysis of diagnostic test accuracy. PLoS One 2014;9:e100182.

11. El Serafy MA, Kassem AM, Omar H, et al. APRI test and hyaluronic acid as non-invasive diagnostic tools for post HCV liver fibrosis: Systematic review and meta-analysis. Arab J Gastroenterol 2017;18:51-7.

12. Lam S, Nettel-Aguirre A, Van Biervliet S, et al. Transient Elastography in the Evaluation of Cystic FibrosisAssociated Liver Disease: Systematic Review and Metaanalysis. J Can Assoc Gastroenterol 2019;2:71-80.

13. Leung DH, Khan M, Minard CG, et al. Aspartate aminotransferase to platelet ratio and fibrosis- 4 as biomarkers in biopsy-validated pediatric cystic fibrosis liver disease. Hepatology 2015;62:1576-83.

14. Calvopina DA, Noble C, Weis A, et al. Supersonic shearwave elastography and APRI for the detection and staging of liver disease in pediatric cystic fibrosis. J Cyst Fibros 2020;19:449-54.

15. Højte C, Jørgensen MH, Jensen F, et al. Extended 
Screening for Cystic Fibrosis-related Liver Disease Including Elastography in Children and Adolescents. J Pediatr Gastroenterol Nutr 2020;71:663-8.

16. Sellers ZM. Barrier to using APRI and GPR as identifiers of cystic fibrosis liver disease. J Cyst Fibros 2021;20:551.

17. Li Q, Song J, Huang Y, et al. The Gamma-GlutamylTranspeptidase to Platelet Ratio Does not Show Advantages than APRI and Fib-4 in Diagnosing Significant Fibrosis and Cirrhosis in Patients With Chronic Hepatitis B: A Retrospective Cohort Study in China. Medicine (Baltimore) 2016;95:e3372.

18. Menten R, Leonard A, Clapuyt P, et al. Transient elastography in patients with cystic fibrosis. Pediatr Radiol 2010;40:1231-5.

19. Levitte S, Lee LW, Isaacson J, et al. Clinical use of shearwave elastography for detecting liver fibrosis in children and adolescents with cystic fibrosis. Pediatr Radiol 2021;51:1369-77.

20. Enaud R, Frison E, Missonnier S, et al. Cystic fibrosis and noninvasive liver fibrosis assessment methods in children. Pediatr Res 2021. [Epub ahead of print]. doi: 10.1038/ s41390-021-01427-4.

21. Kim JR, Suh CH, Yoon HM, et al. The diagnostic performance of shear-wave elastography for liver fibrosis in children and adolescents: A systematic review and diagnostic meta-analysis. Eur Radiol 2018;28:1175-86.

22. Lee SM, Kim MJ, Yoon JH, et al. Comparison of point and 2-dimensional shear wave elastography for the evaluation of liver fibrosis. Ultrasonography 2020;39:288-97.

23. Mărginean CO, Meliţ LE, Ghiga DV, et al. The assessment of liver fibrosis in children with obesity on two methods: transient and two dimensional shear wave elastography. Sci Rep 2019;9:19800.

24. Lewindon PJ, Puertolas-Lopez MV, Ramm LE, et al. Accuracy of Transient Elastography Data Combined With APRI in Detection and Staging of Liver Disease in Pediatric Patients With Cystic Fibrosis. Clin Gastroenterol
Hepatol 2019;17:2561-2569.e5.

25. Sigrist RMS, Liau J, Kaffas AE, et al. Ultrasound Elastography: Review of Techniques and Clinical Applications. Theranostics 2017;7:1303-29.

26. Monti L, Manco M, Lo Zupone C, et al. Acoustic radiation force impulse (ARFI) imaging with Virtual Touch Tissue Quantification in liver disease associated with cystic fibrosis in children. Radiol Med 2012;117:1408-18.

27. Manco M, Zupone CL, Alghisi F, et al. Pilot study on the use of acoustic radiation force impulse imaging in the staging of cystic fibrosis associated liver disease. J Cyst Fibros 2012;11:427-32.

28. Hanley JA, McNeil BJ. A method of comparing the areas under receiver operating characteristic curves derived from the same cases. Radiology 1983;148:839-43.

29. Stonebraker JR, Ooi CY, Pace RG, et al. Features of Severe Liver Disease With Portal Hypertension in Patients With Cystic Fibrosis. Clin Gastroenterol Hepatol 2016;14:1207-1215.e3.

30. Koh C, Sakiani S, Surana P, et al. Adult-onset cystic fibrosis liver disease: Diagnosis and characterization of an underappreciated entity. Hepatology 2017;66:591-601.

31. Karnsakul W, Wasuwanich P, Ingviya T, et al. A longitudinal assessment of non-invasive biomarkers to diagnose and predict cystic fibrosis-associated liver disease. J Cyst Fibros 2020;19:546-52.

32. Gominon AL, Frison E, Hiriart JB, et al. Assessment of Liver Disease Progression in Cystic Fibrosis Using Transient Elastography. J Pediatr Gastroenterol Nutr 2018;66:455-60.

33. Garcovich M, Veraldi S, Di Stasio E, et al. Liver Stiffness in Pediatric Patients with Fatty Liver Disease: Diagnostic Accuracy and Reproducibility of Shear-Wave Elastography. Radiology 2017;283:820-7.

34. Di Serafino M, Severino R, Gioioso M, et al. Paediatric liver ultrasound: a pictorial essay. J Ultrasound 2020;23:87-103.
Cite this article as: Tosco A, Sepe A, Castaldo A, Catzola A, Cimbalo C, Angelini V, Vallone G, Buzzetti R, Raia V, Caprio MG. Non-invasive tools for detection of liver disease in children and adolescents with cystic fibrosis. Transl Pediatr 2021;10(11):2952-2959. doi: 10.21037/tp-21-68 